\title{
Responses of Ber (Zizyphus mauritiana Lamk.) Varieties to Different Level of Salinity
}

\author{
S.S. Verma ${ }^{1}$, R.P. Verma ${ }^{1}$, S.K. Verma ${ }^{1}$, A.L. Yadav ${ }^{1}$ and A.K. Verma ${ }^{2}$ \\ ${ }^{1}$ College of Horticulture and Forestry, NDUAT, Faizabad-224 229, Uttar Pradesh, India \\ ${ }^{2}$ ICAR-National Research Centre on Seed Spices, Ajmer-305206, Rajasthan, India \\ *Corresponding author
}

\section{A B S T R A C T}

\section{Keywords}

Ber, Cultivars, Leaf injury, Mineral composition, Salinity tolerance, Plant growth

Article Info

Accepted:

06 June 2018

Available Online:

10 July 2018
The current work aims to assess response of ber cultivars to different level of salinity. The salinity level $\left(0,4.0,8.0,12.0,16.0 \mathrm{dSm}^{-1} \mathrm{ECe}\right)$ had detrimental effects on growth and mineral composition of transplanted six ber cultivars. High levels of salts tolerated by most of the varieties but severely limit plant growth. High soil salinity decreased the growth (plant height, stem diameter, number of leaves per plant and dry matter yield) and mineral composition of leaves above conductivity of $12 \mathrm{dSm}^{-1}$ ECe. The $\mathrm{Na}^{+}$and $\mathrm{Cl}^{-}$content of leaves were increased with increasing the levels of salinity, whereas $\mathrm{N}, \mathrm{P}, \mathrm{K}^{+}, \mathrm{Ca}^{++}$and $\mathrm{Mg}^{++}$content was decreased. Specific injury due to high accumulation of chloride and sodium in the plant foliage of ber cultivars seemed to be the chief effect of salinity. On the basis of salt tolerance limits, ber cultivars Banarsi Karaka, Narendra Ber Sel.-2 and Ponda can be placed in tolerant and cvs. Narendra Ber Sel.-1 and Gola as semi-tolerant group. This result may help in commercial cultivation of ber in salt pretentious areas.

\section{Introduction}

Ber (Zizyphus mauritiana Lamk.) is a hardy fruit tree belongs to family Rhamnaceae with the chromosome number $2 n=48$. It can be successfully grown in arid and semi-arid zones of Indian states particularly Haryana, Rajasthan, Madhya Pradesh and Gujarat. India has a rich source of underutilized native and exotic fruit trees, which may have a high agro industrial potential and represent an important economic source for the local populations and ber fruit is one of them. It is considered poor man's apple because its fruits are easily available at low cost of production, rich source of vitamin C, protein and minerals (Pareek and Yahia, 2013). The cultivation of ber is gaining popularity in arid and semi-arid region in India because of its low maintenance cost, wide adaptability, low water requirement, high yield, good returns, scope for value addition and suitability even under wastelands (Martinuzzo, 2006). Ber is remunerative fruit, which can be grown successfully even in marginal and saline soils with little care. Due to its hardy nature, adaptability to marginal conditions of soil and climate and the nutritive value of its fruit, it has now become the most 
important fruit of arid regions (Singh and Yadav, 2017).

Salinity affects a large area of the world's arable land making the landscape either barren or unproductive. It is estimated that about $20 \%$ of the earth's land mass and nearly half of the total irrigated land is affected by salinity (Agrawal et al., 2013). Salinity is considered to be a significant factor affecting crop production and agricultural sustainability in many regions of the world and it reduces the value and productivity of the affected lands (Bolarin et al., 1993). Salinity are the major problems in arid and semiarid regions of the country, more prominently in the indus and Gangetic plains in the north. The process of salinization involves accumulation of soluble salts on the soil surface. High concentration of salt in the root zone reduces soil water potential and the availability of water (Arndt et al., 2000). As a result of this, reduction of the water content leading to dehydration at cellular level and osmotic stress is observed. The increased amount of sodium $\left(\mathrm{Na}^{+}\right)$and chloride $\left(\mathrm{Cl}^{-}\right)$in the environment affects the uptake of many indispensable nutrients through competitive interactions and by affecting the ion selectivity of membranes (Shiyab et al., 2013). Salt stress is a complex physico chemical progression, in which several biological molecules such as nucleic acids, proteins, carbohydrates, lipids, hormones, ions, free radicals, free amino acids and mineral elements are actively involved (Munns, 2002; Liu and Baird, 2004; Verma et al., 2018). Besides this, salt stress decrease the photosynthesis (Pradeep and Jumbhale, 2000), causes stomata closure (Hernandez and Almansa, 2002) and increases the concentration of activated oxygen species (AOS), such as superoxide radical $\left(\mathrm{O}^{-}\right)$, hydrogen peroxide $\left(\mathrm{H}_{2} \mathrm{O}_{2}\right)$ and hydroxyl radical $\left(\mathrm{OH}^{-}\right)$via enhanced leakage of electrons to oxygen (Arzani, 2008). The salinity tolerance of several fruit crops has been studied by various workers, but no information regarding salinity tolerance of ber is available. In view of these facts it was considered necessary to initiate investigation to study the salinity tolerance of Zizyphus mauritiana species of ber. The present report is an attempt to evaluate the effect of soil salinity on plant growth and mineral composition of six ber cultivars.

\section{Materials and Methods}

The investigations were undertaken at the experimental orchard of the Department of Horticulture, Narendra Deva University of Agriculture and Technology, Faizabad, Uttar Pradesh (India) during the year (2012-2013) with six ber cultivars in cement pots $(45 \times 45 \times 45 \mathrm{~cm}$ size $)$ containing sandy loam soil. The treatments included five level of salinity $\left(0,4.0,8.0,12.0,16.0 \mathrm{dSm}^{-1} \mathrm{ECe}\right)$. The salinity levels were created by adding differential amounts of $\mathrm{NaCl}, \mathrm{Na}_{2} \mathrm{SO}_{4}, \mathrm{CaCl}_{2}$ and $\mathrm{MgCl}_{2} \cdot 6 \mathrm{H}_{2} \mathrm{O}$ in the soil in such proportions, so as to obtain the $\mathrm{Na}: \mathrm{Ca}+\mathrm{Mg}$ ratio of $1: 1$ and $1: 2$, respectively. Salt solution containing above salts was prepared on the basis of saturation percentage of the soil. The soil thus treated was covered with polythene sheet for a two week to allow uniform distribution of salts. The salts solution was thoroughly sprayed with a fine nozzle sprayer. The soil was analysed for ECe before transplanting of ber cultivars. The observed salinity levels along with their designations are given in Table 1 .

Three months old budded ber plants of uniform size were transplanted in each pot. Only one budded plants was allowed to grow in each pot. After 30 days of transplanting on the basis of visual observation and seeing the moisture conditions of the soil, the pots were irrigated with canal water. The irrigation was applied through a rose cane alternatively to facilitate the uniform distribution of salts, 
there by maintaining the particular salinity in the root zone. The recommended cultural practices were followed throughout the growth period of the ber cultivars.

Observation on growth characters viz plant height, stem diameter at $15 \mathrm{~cm}$ above ground level and number of leaves per plant at monthly interval were recorded. Dry matter yield of shoots and roots were taken at the time of uprooting the plants, before harvesting plants, leaf samples for mineral analysis were obtained by collecting mature representative leaves from each plant. Leaves were washed with distilled water dried in an oven at $70^{\circ} \mathrm{C}$ and ground finally. Form each sample $0.5 \mathrm{~g}$ of material was digested in the diacid mixture (4:1 $\left.\mathrm{HNO}_{3} ; \mathrm{HClO}_{4}\right)$ and $\mathrm{N}$ was analysed with the help of micro Kjeldahl flask. For determining $\mathrm{Ca}^{++}$and $\mathrm{Mg}^{++}$were determined with Atomic Absorption Spectrophotometry (AA); while $\mathrm{K}^{+}$and $\mathrm{Na}^{+}$was estimated with flame emission, Chloride $\left(\mathrm{Cl}^{-}\right)$in plant tissue extracts was determined by potentiometric titration. The procedure involves extraction of $\mathrm{Cl}^{-}$from dried, powdered plant material in hot deionized water. The concentrations were determined directly by silver ion titration using Eppendorf chloridometer (type 6610). The Experiment was laid out in Completely Randomized Design (CRD). Total twenty four treatment combustions were used and each treatment was replicated three times. The data were evaluated with analysis of variance (ANOVA).

\section{Results and Discussion}

\section{Visible symptoms of salt injury}

The plants grown in normal soil shows that no injury symptoms were noticed in all varieties of ber (Table 2), even though increasing the salinity level up to the $8 \mathrm{dS} / \mathrm{m}$ no injury symptoms (A) were shown. Further increase in the salinity level from $8 \mathrm{dS} / \mathrm{m}$ to $12 \mathrm{dS} / \mathrm{m}$ there was injury symptoms in the form of changes in the leaves colour (B) and burning and necrosis of apical portion of leaves (C) were observed. Ber variety Narendra Ber Sel.3 shows $\mathrm{B}$ and $\mathrm{C}$ symptoms. Varieties Narendra Ber Sel.-1, Ponda and Gola showed $B$ types of injury while Banarsi Karaka and Narendra Ber Sel.-2 did not show any types of symptoms (A) at the salinity level $12 \mathrm{dS} / \mathrm{m}$. When the salinity increased from 12 to 16 $\mathrm{dS} / \mathrm{m}$, all varieties showed injury symptoms (B and C). Varieties Banarsi Karaka and Narendra Ber Sel.-2 showed B types salinity, while Narendra Ber Sel.-1, Narendra Ber Sel.3, Ponda and Gola shoes BC types of symptoms. Similar results were recorded by Singh et al., (2018 a, b) in guava (Psidium guajava) and bael (Aegle marmelos) under shallow saline watertable conditions.

\section{Effect of salinity on plant growth}

A glance of results on effect of salt stress revealed that higher concentration of soluble salts in the soil had an adverse effect on the growth of ber cultivars (Table 3, 4, 5, 6 and 7). The high levels of soil salinity decreased the plant height of different cultivars of ber (Table $3)$. In the normal soil the plant height was highest $(157.65 \mathrm{~cm})$ in Narendra Ber Sel.-2 cultivar followed by Banarsi Karaka (155.24 $\mathrm{cm})$ and Gola $(152.38 \mathrm{~cm})$. The lowest (133.78) plant height was recoded in Narendra Ber Sel.-1. When the different concentrations of salts added in the soil the plant height of different cultivars were decreased and lowest plant height was recorded at highest salinity level $(16 \mathrm{dS} / \mathrm{m})$. Among the different cultivars at highest salinity level (16 dS/m), maximum plant height was recorded in Banarsi Karaka $(94.26 \mathrm{~cm})$ followed by Narendra Ber Sel.-2 $(91.83 \mathrm{~cm})$ which were statistically at par the each other. Lowest $(76.19 \mathrm{~cm})$ plant height was observed in Narendra Ber Sel.-1. Data presented in Table 4 revealed that stem diameter deceases with the increased in the 
salt concentrations in all tested varieties of ber. Highest $(1.11 \mathrm{~cm})$ diameter was recorded in Banarsi Karaka followed by Narendra Ber Sel.-2 $(1.07 \mathrm{~cm})$ and Ponda $(1.04 \mathrm{~cm})$ at salinity level $16 \mathrm{dS} / \mathrm{m}$. Likewise, Table 5 shows that at the highest salinity level (16 $\mathrm{dS} / \mathrm{m}$ ), highest number of leaves per plant (151) was recorded in Banarsi Karaka followed by Narendra Ber Sel.-2 (140) and Ponda (138), while the minimum number of leavers per plant was recorded in Gola (110). Salinity levels affect the dry weight of shoot in all the ber varieties (Table 6), height was recorded in Banarsi Karaka (124.67 g) followed by Narendra Ber Sel.-2 (119.80 g) and Ponda (111.90 g), while the least dry weight war observed in Narendra Ber Sel.-3 $(106.70 \mathrm{~g})$ and Gola $(106.30 \mathrm{~g})$. Similar trend were also observed with regards to dry weight of roots (Table 7), maximum (58.50 g) dry root weight was recorded in Banarsi Karaka, while minimum was in Gola (48.80 g).

Decreasing in plant growth is normally observed under saline conditions. Hokmalipour (2015) found that increasing salt concentrations $(0,2,4,6,8$, and $10 \mathrm{dS} \mathrm{m}^{-1}$ ) significantly decreased the seedling height, shoot and root lengths, seed germination percentage, germination rate, fresh, and dry seedling weight and seed vigor index in fennel and cumin. In another studied done on guava and bael by Singh et al., (2018 $\mathrm{a}, \mathrm{b}$ ) found that in saline soils, plant growth is adversely affected initially by the osmotic stress and subsequently by specific ion toxicities. Nonetheless, marked genotypic differences exist even in highly salt sensitive crops like citrus where different scion and rootstock cultivars greatly vary with each other in salt-induced growth reduction (Singh et al., 2015). In bael, Shukla and Singh (1996) reported that salinized bael plants show excessive uptake of $\mathrm{Na}^{+}$ions and reduced tissue concentrations of $\mathrm{K}^{+}$and $\mathrm{Ca}^{2+}$ resulting in metabolic impairments, decrease in photosynthetic assimilation and the eventual appearance of injury symptoms. At $6.5 \mathrm{dS} / \mathrm{m}$ salinity, only about one thirds of the leaves showed salt injury symptoms in bael cultivar NB-5 while nearly two thirds of the leaves were severely affected in NB-9 and CB-2 plants (Singh et al., 2016). Similar observations have been recorded by other workers such as Rao and Singh (2004) in aonla; Awasthi et al., (1995) and Shen et al., (2009) in ber. The inhibitory effect of salinity on plant growth may probably due to a) osmotic effect on plant roots and thus causing physiological scarcity of water b) the toxic accumulation of salinity ions $(\mathrm{Cl}$ and $\mathrm{Na}$ ) in plant tissue and c) nutrient imbalance due to excess of salinity ions in soil and plant tissues. But in fruit trees chloride toxicity has been reported as the chief effect of salinity (Chartzoulakis, 2005).

\section{Effects on chlorine content (\%)}

In chloride dominated saline soils which were used for the experimentation the first injury symptom was the inhibition of plant growth followed by change in colour of leaves. Perusals of data presented in Table 8 reveals that increase level of salinity enhance the accumulation of chloride by the plants. The highest $(0.93 \%)$ chloride accumulation was recorded in Narendra Ber Sel.-1 followed by Narendra Ber Sel.-2 (0.91\%), while the minimum $(0.79 \%)$ chloride accumulation was recorded in Banarsi Karaka at $16 \mathrm{dS} / \mathrm{m}$. The increase in the leaf concentration of chloride seems to be fairly correlating with plant growth. The chloride content of foliage increased with the increasing levels of salinity and increase was more marked at and above the salinity level of $12 \mathrm{~d} \mathrm{Sm}^{-1} \mathrm{ECe}$ per cent corresponding to $8 \mathrm{dSm}^{-1} \mathrm{ECe}$ does not seem to have more adverse effect on plant growth but on increase above this level of chloride accumulation had an adverse effect on plant growth. 
Table.1 Showing desired and obtained salinity levels

\begin{tabular}{|c|c|c|}
\hline Desired ECe level & Initial level \\
\hline & ECe $\left.\mathbf{d S m}^{-\mathbf{1}}\right)$ & $\mathbf{p H}$ \\
\hline Control $(\mathbf{0})$ & 0.81 & 7.3 \\
\hline $\mathbf{4}$ & 3.98 & 7.5 \\
\hline $\mathbf{8}$ & 8.10 & 7.6 \\
\hline $\mathbf{1 2}$ & 11.93 & 7.7 \\
\hline $\mathbf{1 6}$ & 15.67 & 8.0 \\
\hline
\end{tabular}

Table.2 Effect of salinity levels on leaf injury in relation to salinity of ber cultivars

\begin{tabular}{|l|c|c|c|c|c|}
\hline \multirow{2}{*}{ Cultivar } & \multicolumn{5}{|c|}{ Soil Salinity level (ECe) } \\
\cline { 2 - 6 } & Normal Soil & $\mathbf{4}$ & $\mathbf{8}$ & $\mathbf{1 2}$ & $\mathbf{1 6}$ \\
\hline Banarsi Karaka & A & A & A & A & B \\
\hline Narendra Ber Sel.-1 & A & A & A & B & B C \\
\hline Narendra Ber Sel.-2 & A & A & A & A & B C \\
\hline Narendra Ber Sel.-3 & A & A & A & B C & B C \\
\hline Ponda & A & A & A & B & B C \\
\hline Gola & A & A & A & B & \\
\hline $\begin{array}{l}\text { A. No symptoms appeared, B. Change the leaves colour from dark green to yellowish or whitish green, C. Burning and necrosis of } \\
\text { apical portion leaves }\end{array}$
\end{tabular}

Table.3 Effect of salinity levels on plant height $(\mathrm{cm})$ of ber cultivars

\begin{tabular}{|l|c|c|c|c|c|c|}
\hline \multirow{2}{*}{ Cultivar } & \multicolumn{5}{|c|}{ Soil salinity level (ECe) } & \multirow{2}{*}{ Mean } \\
\cline { 2 - 7 } & Normal Soil & $\mathbf{4}$ & $\mathbf{8}$ & $\mathbf{1 2}$ & $\mathbf{1 6}$ & \\
\hline Banarsi Karaka & 155.24 & 156.80 & 152.85 & 112.02 & 94.26 & 134.23 \\
\hline Narendra Ber Sel.-1 & 133.78 & 134.23 & 113.25 & 94.23 & 76.19 & 110.34 \\
\hline Narendra Ber Sel.-2 & 157.65 & 157.57 & 138.79 & 120.94 & 91.83 & 133.36 \\
\hline Narendra Ber Sel.-3 & 142.25 & 138.16 & 119.15 & 97.61 & 79.46 & 115.33 \\
\hline Ponda & 144.30 & 139.85 & 129.85 & 106.22 & 83.51 & 120.75 \\
\hline Gola & 152.38 & 146.01 & 127.54 & 103.34 & 79.41 & 121.74 \\
\hline Mean & 147.60 & 145.44 & 13.24 & 105.73 & 84.11 & \\
\hline & Cultivar (C) & \multicolumn{3}{|c|}{ ECe Level (S) } & Interaction (C X S) \\
\hline C.D. (P=0.05) & \multicolumn{4}{|c|}{4.05} & \multicolumn{4}{c|}{9.05} \\
\hline
\end{tabular}

Table.4 Effect of salinity levels on stem diameter $(\mathrm{cm})$ of ber cultivars

\begin{tabular}{|c|c|c|c|c|c|c|}
\hline \multirow[t]{2}{*}{ Cultivar } & \multicolumn{5}{|c|}{ Soil salinity level (ECe) } & \multirow[t]{2}{*}{ Mean } \\
\hline & Normal Soil & 4 & 8 & 12 & 16 & \\
\hline Banarsi Karaka & 1.98 & 1.92 & 1.74 & 1.39 & 1.11 & 1.63 \\
\hline Narendra Ber Sel.-1 & 1.82 & 1.86 & 1.60 & 1.30 & 1.00 & 1.52 \\
\hline Narendra Ber Sel.-2 & 1.98 & 1.97 & 1.60 & 1.29 & 1.07 & 1.58 \\
\hline Narendra Ber Sel.-3 & 1.86 & 1.86 & 1.71 & 1.34 & 1.00 & 1.55 \\
\hline Ponda & 1.91 & 1.95 & 1.68 & 1.22 & 1.04 & 1.56 \\
\hline Gola & 1.77 & 1.64 & 1.52 & 1.22 & 1.01 & 1.43 \\
\hline \multirow[t]{2}{*}{ Mean } & 1.89 & 1.87 & 1.64 & 1.29 & 1.04 & \\
\hline & \multicolumn{2}{|c|}{ Cultivar (C) } & \multicolumn{2}{|c|}{ ECe Level (S) } & \multicolumn{2}{|c|}{ Interaction (C X S) } \\
\hline C.D. $(P=0.05)$ & \multicolumn{2}{|c|}{0.03} & \multicolumn{2}{|c|}{0.03} & \multicolumn{2}{|c|}{0.08} \\
\hline
\end{tabular}


Table.5 Effect of salinity levels on number of leaves/plant of ber cultivars

\begin{tabular}{|l|l|l|l|l|l|l|}
\hline \multirow{2}{*}{ Cultivar } & \multicolumn{5}{|c|}{ Soil salinity level (ECe) } & \multirow{2}{*}{ Mean } \\
\cline { 2 - 7 } & Normal Soil & $\mathbf{4}$ & $\mathbf{8}$ & $\mathbf{1 2}$ & $\mathbf{1 6}$ & \\
\hline Banarsi Karaka & 292.33 & 291.67 & 257.00 & 189.00 & 151.00 & 236.20 \\
\hline Narendra Ber Sel.-1 & 233.33 & 225.00 & 194.67 & 143.00 & 114.33 & 182.07 \\
\hline Narendra Ber Sel.-2 & 301.33 & 290.33 & 253.67 & 188.33 & 140.67 & 234.87 \\
\hline Narendra Ber Sel.-3 & 245.33 & 239.33 & 190.67 & 160.33 & 131.33 & 193.40 \\
\hline Ponda & 263.00 & 262.00 & 238.00 & 178.00 & 138.00 & 215.80 \\
\hline Gola & 245.33 & 241.33 & 189.00 & 167.00 & 110.00 & 190.53 \\
\hline Mean & 263.44 & 258.28 & 220.50 & 170.94 & 130.89 & \\
\hline & Cultivar (C) & \multicolumn{5}{|c|}{ ECe Level (S) } \\
\hline C.D. $(\mathbf{P = 0 . 0 5 )}$ & 5.51 & 5.03 & Interaction (C X S) \\
\hline
\end{tabular}

Table.6 Effect of salinity levels on dry weight of shoots (g) of ber cultivars

\begin{tabular}{|l|c|c|c|c|c|c|}
\hline \multirow{2}{*}{ Cultivar } & \multicolumn{5}{|c|}{ Soil salinity level (ECe) } & \multirow{2}{*}{ Mean } \\
\cline { 2 - 7 } & Normal Soil & $\mathbf{4}$ & $\mathbf{8}$ & $\mathbf{1 2}$ & $\mathbf{1 6}$ & \\
\hline Banarsi Karaka & 184.00 & 176.33 & 177.90 & 151.80 & 124.67 & 162.94 \\
\hline Narendra Ber Sel.-1 & 170.30 & 164.47 & 146.20 & 125.50 & 118.00 & 144.89 \\
\hline Narendra Ber Sel.-2 & 181.30 & 178.50 & 159.53 & 143.23 & 119.80 & 156.47 \\
\hline Narendra Ber Sel.-3 & 163.03 & 159.90 & 143.87 & 122.07 & 106.30 & 139.03 \\
\hline Ponda & 167.90 & 163.90 & 146.80 & 131.77 & 111.90 & 144.45 \\
\hline Gola & 157.97 & 154.07 & 140.07 & 123.03 & 106.70 & 136.37 \\
\hline Mean & 170.75 & 166.19 & 152.40 & 132.90 & 114.56 & \\
\hline & Cultivar (C) & \multicolumn{3}{|c|}{ ECe Level (S) } & Interaction (C X S) \\
\hline C.D. (P=0.05) & \multicolumn{2}{|c|}{2.03} & \multicolumn{3}{|c|}{4.55} \\
\hline
\end{tabular}

Table.7 Effect of salinity levels on dry weight of roots (g) of ber cultivars

\begin{tabular}{|c|c|c|c|c|c|c|}
\hline \multirow[t]{2}{*}{ Cultivar } & \multicolumn{5}{|c|}{ Soil salinity level (ECe) } & \multirow[t]{2}{*}{ Mean } \\
\hline & Normal Soil & 4 & 8 & 12 & 16 & \\
\hline Banarsi Karaka & 98.30 & 99.20 & 82.93 & 73.47 & 58.50 & 82.48 \\
\hline Narendra Ber Sel.-1 & 90.73 & 85.53 & 75.40 & 65.10 & 49.70 & 73.29 \\
\hline Narendra Ber Sel.-2 & 99.53 & 99.17 & 82.43 & 70.80 & 56.17 & 81.62 \\
\hline Narendra Ber Sel.-3 & 90.37 & 90.88 & 78.50 & 66.23 & 54.83 & 76.16 \\
\hline Ponda & 93.33 & 91.93 & 80.33 & 66.80 & 55.27 & 77.53 \\
\hline Gola & 94.00 & 88.83 & 76.50 & 66.37 & 48.80 & 74.90 \\
\hline \multirow[t]{2}{*}{ Mean } & 94.38 & 92.59 & 79.35 & 68.13 & 53.88 & \\
\hline & \multicolumn{2}{|c|}{ Cultivar (C) } & \multicolumn{2}{|c|}{ ECe Level (S) } & \multicolumn{2}{|c|}{ Interaction (C X S) } \\
\hline C.D. $(P=0.05)$ & \multicolumn{2}{|c|}{1.37} & \multicolumn{2}{|c|}{1.25} & \multicolumn{2}{|c|}{3.07} \\
\hline
\end{tabular}


Table.8 Effect of salinity levels on chlorine content in leaves (\%) of ber leaves in different cultivars

\begin{tabular}{|c|c|c|c|c|c|c|}
\hline \multirow[t]{2}{*}{ Cultivar } & \multicolumn{5}{|c|}{ Soil Salinity level (ECe) } & \multirow[t]{2}{*}{ Mean } \\
\hline & Normal Soil & 4 & 8 & 12 & 16 & \\
\hline Banarsi Karaka & 0.25 & 0.30 & 0.36 & 0.56 & 0.79 & 0.45 \\
\hline Narendra Ber Sel.-1 & 0.25 & 0.32 & 0.47 & 0.63 & 0.93 & 0.52 \\
\hline Narendra Ber Sel.-2 & 0.21 & 0.25 & 0.38 & 0.58 & 0.91 & 0.46 \\
\hline Narendra Ber Sel.-3 & 0.24 & 0.27 & 0.41 & 0.62 & 0.86 & 0.48 \\
\hline Ponda & 0.23 & 0.28 & 0.40 & 0.59 & 0.82 & 0.46 \\
\hline Gola & 0.28 & 0.30 & 0.38 & 0.56 & 0.85 & 0.47 \\
\hline \multirow[t]{2}{*}{ Mean } & 0.24 & 0.28 & 0.40 & 0.59 & 0.86 & \\
\hline & \multicolumn{2}{|c|}{ Cultivar (C) } & \multicolumn{2}{|c|}{ ECe Level (S) } & \multicolumn{2}{|c|}{ Interaction (C X S) } \\
\hline C.D. $(P=0.05)$ & \multicolumn{2}{|c|}{0.016} & \multicolumn{2}{|c|}{0.0 .14} & \multicolumn{2}{|c|}{0.035} \\
\hline
\end{tabular}

Table.9 Effect of salinity levels on N, P and K composition of ber leaves in different cultivars (dry weight basis)

\begin{tabular}{|c|c|c|c|c|c|c|c|c|c|c|c|c|c|c|c|c|c|c|}
\hline \multirow[t]{3}{*}{ Cultivar } & \multicolumn{6}{|c|}{ Nitrogen (\%) } & \multicolumn{6}{|c|}{ Phosphorus (\%) } & \multicolumn{6}{|c|}{ Potassium (\%) } \\
\hline & \multicolumn{5}{|c|}{ Soil Salinity level } & \multirow[t]{2}{*}{ Mean } & \multicolumn{5}{|c|}{ Soil Salinity level } & \multirow[t]{2}{*}{ Mean } & \multicolumn{5}{|c|}{ Soil Salinity level } & \multirow[t]{2}{*}{ Mean } \\
\hline & Normal Soil & 4 & 8 & 12 & 16 & & Normal Soil & 4 & 8 & 12 & 16 & & Normal Soil & 4 & 8 & 12 & 16 & \\
\hline Banarsi Karaka & 4.28 & 4.24 & 4.17 & 3.95 & 3.66 & 4.06 & 0.013 & 0.012 & 0.029 & 0.013 & 0.012 & 0.029 & 0.85 & 0.83 & 0.79 & 0.74 & 0.66 & 0.77 \\
\hline $\begin{array}{l}\text { Narendra Ber } \\
\text { Sel.-1 }\end{array}$ & 4.15 & 4.14 & 4.01 & 3.62 & 3.29 & 3.84 & 0.03 & 0.03 & 0.08 & 0.03 & 0.03 & 0.08 & 0.74 & 0.74 & 0.71 & 0.65 & 0.46 & 0.66 \\
\hline $\begin{array}{l}\text { Narendra Ber } \\
\quad \text { Sel.-2 }\end{array}$ & 4.24 & 4.26 & 4.10 & 3.89 & 3.54 & 4.01 & 0.013 & 0.012 & 0.029 & 0.013 & 0.012 & 0.029 & 0.83 & 0.80 & 0.76 & 0.70 & 0.59 & 0.74 \\
\hline $\begin{array}{l}\text { Narendra Ber } \\
\quad \text { Sel.-3 }\end{array}$ & 4.11 & 4.13 & 3.92 & 3.73 & 3.38 & 3.85 & 0.03 & 0.03 & 0.08 & 0.03 & 0.03 & 0.08 & 0.79 & 0.74 & 0.73 & 0.60 & 0.43 & 0.66 \\
\hline Ponda & 4.19 & 4.17 & 3.99 & 3.82 & 3.47 & 3.93 & 0.013 & 0.012 & 0.029 & 0.013 & 0.012 & 0.029 & 0.80 & 0.79 & 0.73 & 0.68 & 0.52 & 0.70 \\
\hline Gola & 4.17 & 4.13 & 4.00 & 3.72 & 3.39 & 3.88 & 0.03 & 0.03 & 0.08 & 0.03 & 0.03 & 0.08 & 0.79 & 0.73 & 0.70 & 0.60 & 0.48 & 0.66 \\
\hline \multirow[t]{2}{*}{ Mean } & 4.19 & 4.18 & 4.03 & 3.79 & 3.45 & & 0.013 & 0.012 & 0.029 & 0.013 & 0.012 & & 0.80 & 0.77 & 0.74 & 0.66 & 0.52 & \\
\hline & \multicolumn{2}{|c|}{ Cultivar (C) } & \multicolumn{2}{|c|}{$\begin{array}{l}\text { ECe Level } \\
\quad \text { (S) }\end{array}$} & \multicolumn{2}{|c|}{$\begin{array}{l}\text { Interaction } \\
\quad(\mathrm{C} \times \mathrm{S})\end{array}$} & \multicolumn{2}{|c|}{ Cultivar (C) } & \multicolumn{2}{|c|}{ ECe Level (S) } & \multicolumn{2}{|c|}{$\begin{array}{c}\text { Interaction }(C \\
\text { X S })\end{array}$} & \multicolumn{3}{|c|}{ Cultivar (C) } & \multicolumn{2}{|c|}{$\begin{array}{l}\text { ECe Level } \\
\quad \text { (S) }\end{array}$} & $\begin{array}{c}\text { Interaction } \\
\text { (C X S) }\end{array}$ \\
\hline C.D. $(P=0.05)$ & \multicolumn{2}{|l|}{0.03} & \multicolumn{2}{|c|}{0.03} & \multicolumn{2}{|c|}{0.08} & \multicolumn{2}{|l|}{0.03} & \multicolumn{2}{|c|}{0.03} & \multicolumn{2}{|c|}{0.08} & \multicolumn{2}{|c|}{0.013} & \multicolumn{3}{|c|}{0.012} & 0.030 \\
\hline
\end{tabular}


Table.10 Effect of salinity levels on $\mathrm{Na}^{+}, \mathrm{Ca}^{++}$and $\mathrm{Mg}^{++}$composition of ber leaves in different cultivars (dry weight basis)

\begin{tabular}{|c|c|c|c|c|c|c|c|c|c|c|c|c|c|c|c|c|c|c|}
\hline \multirow[t]{3}{*}{ Cultivar } & \multicolumn{6}{|c|}{ Sodium (\%) } & \multicolumn{6}{|c|}{ Calcium (\%) } & \multicolumn{6}{|c|}{ Magnesium (\%) } \\
\hline & \multicolumn{5}{|c|}{ Soil Salinity level } & \multirow[t]{2}{*}{ Mean } & \multicolumn{5}{|c|}{ Soil Salinity level } & \multirow[t]{2}{*}{ Mean } & \multicolumn{5}{|c|}{ Soil Salinity levels } & \multirow[t]{2}{*}{ Mean } \\
\hline & $\begin{array}{l}\text { Normal } \\
\text { Soil }\end{array}$ & 4 & 8 & 12 & 16 & & $\begin{array}{l}\text { Normal } \\
\text { Soil }\end{array}$ & 4 & 8 & 12 & 16 & & $\begin{array}{l}\text { Normal } \\
\text { Soil }\end{array}$ & 4 & 8 & 12 & 16 & \\
\hline Banarsi Karaka & 0.36 & 0.37 & 0.42 & 0.49 & 0.61 & 0.45 & 2.56 & 2.57 & 2.47 & 2.34 & 2.07 & 2.40 & 0.83 & 0.78 & 0.72 & 0.65 & 0.53 & 0.70 \\
\hline $\begin{array}{c}\text { Narendra Ber } \\
\text { Sel.-1 }\end{array}$ & 0.38 & 0.39 & 0.44 & 0.51 & 0.60 & 0.46 & 2.36 & 2.42 & 2.34 & 2.21 & 1.85 & 2.24 & 0.69 & 0.66 & 0.62 & 0.55 & 0.42 & 0.59 \\
\hline $\begin{array}{c}\text { Narendra Ber } \\
\text { Sel.-2 }\end{array}$ & 0.40 & 0.40 & 0.44 & 0.53 & 0.60 & 0.47 & 2.52 & 2.53 & 2.42 & 2.23 & 1.92 & 2.33 & 0.77 & 0.72 & 0.69 & 0.59 & 0.47 & 0.65 \\
\hline $\begin{array}{l}\text { Narendra Ber } \\
\text { Sel.-3 }\end{array}$ & 0.41 & 0.40 & 0.47 & 0.54 & 0.62 & 0.49 & 2.36 & 2.37 & 2.28 & 2.19 & 1.86 & 2.21 & 0.67 & 0.67 & 0.63 & 0.54 & 0.40 & 0.58 \\
\hline Ponda & 0.39 & 0.38 & 0.45 & 0.53 & 0.59 & 0.47 & 2.41 & 2.41 & 2.41 & 2.22 & 1.92 & 2.27 & 0.71 & 0.70 & 0.66 & 0.57 & 0.43 & 0.62 \\
\hline Gola & 0.40 & 0.41 & 0.47 & 0.57 & 0.65 & 0.50 & 2.34 & 2.39 & 2.26 & 2.14 & 1.83 & 2.19 & 0.71 & 0.64 & 0.61 & 0.52 & 0.39 & 0.57 \\
\hline \multirow[t]{2}{*}{ Mean } & 0.39 & 0.39 & 0.45 & 0.53 & 0.61 & & 2.43 & 2.45 & 2.37 & 2.22 & 1.91 & & 0.73 & 0.70 & 0.66 & 0.57 & 0.44 & \\
\hline & \multicolumn{2}{|c|}{ Cultivar (C) } & \multicolumn{2}{|c|}{$\begin{array}{l}\text { ECe Level } \\
\text { (S) }\end{array}$} & \multicolumn{2}{|c|}{$\begin{array}{l}\text { Interaction } \\
\text { (C X S) }\end{array}$} & \multicolumn{2}{|c|}{ Cultivar (C) } & \multicolumn{2}{|c|}{$\begin{array}{c}\text { ECe Level } \\
\text { (S) }\end{array}$} & \multicolumn{2}{|c|}{$\begin{array}{l}\text { Interaction } \\
\quad(\mathrm{C} X \mathrm{~S})\end{array}$} & \multicolumn{2}{|c|}{ Cultivar (C) } & \multicolumn{2}{|c|}{$\begin{array}{c}\text { ECe Level } \\
\text { (S) }\end{array}$} & \multicolumn{2}{|c|}{$\begin{array}{c}\text { Interaction } \\
\text { (C X S) }\end{array}$} \\
\hline C.D. $(P=0.05)$ & \multicolumn{2}{|c|}{0.015} & \multicolumn{2}{|c|}{0.014} & \multicolumn{2}{|c|}{0.034} & \multicolumn{2}{|c|}{0.029} & \multicolumn{2}{|c|}{0.026} & \multicolumn{2}{|c|}{0.064} & \multicolumn{2}{|c|}{0.013} & \multicolumn{2}{|c|}{0.012} & \multicolumn{2}{|c|}{0.029} \\
\hline
\end{tabular}


In the higher level of chlorine content in the plants, the general yellowing, marginal and tip burning were observed which progressed towards the mid rib and base of leaves. Severely injured leaves become dry and abscised. Such type of leaf injury might be due to high osmotic pressure of salt substrate, which inhibits the uptake of water by the plant roots on the other hand; this may be due to accumulation of chloride $\left(\mathrm{Cl}^{-}\right)$ions in plants at toxic levels. The findings on plant chloride content in ber cultivars were similar to the findings reported by Agrawal et al., (2013) and Sherani et al., (2017) in Ziziphus mauritiana; Al-Absi et al., (2003) in Olive europaea; Rao and Singh (2004) in Emblica officinalis.

\section{Effects on mineral constituents}

Data presented in Table 9 and 10 shows that soil salinity affected the mineral constituents of the leaves differently. The concentration of all the salinity ions $\left(\mathrm{Na}^{+}\right.$and $\left.\mathrm{Cl}^{-}\right)$in the leaves of different ber cultivars increased where as $\mathrm{N}, \mathrm{P}, \mathrm{K}^{+}, \mathrm{Ca}^{++}$and $\mathrm{Mg}^{++}$decreased with increasing level of salinity (from 4 to 16 $\mathrm{dS} / \mathrm{m})$. The salt stress decreases the N,P and $\mathrm{K}^{+}$content in the ber leaves, as he salinity increases in all varieties of ber (Table 8). In normal soil, maximum $(4.28 \%)$ nitrogen content was recorded in Banarsi Karaka while applying salt stress the nitrogen content reduced to $3.66 \%$ at $16 \mathrm{dS} / \mathrm{m}$. Similarly, $\mathrm{P}$ and $\mathrm{K}^{+}$content were recorded highest in Banarsi Karaka i.e. $0.012 \%$ and $0.66 \%$, respectively. Lowest level $(0.43 \%)$ of $\mathrm{K}^{+}$was recorded in Gola and Narendra Ber Sel.-1. The sodium accumulation showed an increase with increasing levels of salinity (from 4 to 16 $\mathrm{dS} / \mathrm{m})$ while $\mathrm{Ca}^{++}$and $\mathrm{Mg}^{++}$showed decreasing trend (Table 10). The The $\mathrm{Na}^{+}$ content was increased with increase in the salinity level and recorded maximum (0.65) at $16 \mathrm{dS} / \mathrm{m}$ in Gola followed by Narendra Ber Sel.-3 (0.62\%), Banarsi Karaka (0.61\%),
Narendra Ber Sel.-1 and $2(0.60 \%)$ and Ponda $(0.59 \%)$. In contrast with $\mathrm{Na}^{+}, \mathrm{Ca}^{++}$and $\mathrm{Mg}^{++}$ were deceased with increase in the salinity level. Maximum $(2.07 \%) \mathrm{Ca}^{++}$content were recorded in Banarsi Karaka while, lowest were recorded in Gola (1.83\%). Similar results also recorded with respect to $\mathrm{Mg}^{++}$ content, maximum $\mathrm{Mg}^{++}$content were recorded in Banarsi Karaka (0.70\%), whereas, lowest were recorded in Gola $(0.57 \%)$.

Plants require different macro and micronutrients for metabolic activities such as photosynthesis and the production of secondary metabolites. Salinity-induced decrease in soil osmotic potential and increased concentrations of toxic ions $\left(\mathrm{Na}^{+}\right.$ and $\mathrm{Cl}^{-}$) restrain the uptake of water and essential nutrients by plants. Salinity almost invariably leads to reduced $\mathrm{N}$ availability in plants (Feigin, 1985). Similarly, in most of the cases, salt-stressed plants show deficient $\mathrm{P}$ levels with adverse consequences for photosynthesis and other energy-dependent growth processes (Overlach et al., 1993). Elevated $\mathrm{Na}^{+}$levels in growing medium usually suppress $\mathrm{K}^{+}$supply to plants. $\mathrm{K}^{+}$not only acts as an essential cofactor for many enzymes but also plays critical roles in cellular osmotic balance and stomatal regulation (Munns, 2005). Available evidence suggests that some crop genotypes tend to preferentially accumulate $\mathrm{K}^{+}$to partly overcome $\mathrm{Na}^{+}$toxicity (Baatour, et al., 2010). In majority of the crop plants, salinity lowers $\mathrm{Ca}^{++}$levels leading to membrane permeability, EL and other harmful effects (Ashraf et al., 2004). A decreased in the concentration of all the primary and secondary nutrients in leaves of ber cultivars viz, $\mathrm{N}, \mathrm{P}, \mathrm{K}^{+}, \mathrm{Ca}^{++}$and $\mathrm{Mg}^{++}$in the foliage with increasing levels of salinity in different plants may be due to their presence of high concentration of salinity ions in the soil. A decrease in $\mathrm{N}, \mathrm{P}$ and $\mathrm{K}^{+}$content of foliage has also been reported (Awasthi, 1995; Shukla 
and Singh, 1996). Same decreasing trend in $\mathrm{Ca}^{++}$and $\mathrm{Mg}^{++}$was reported by $\mathrm{Al}-$ Abdoulhadi et al., $(2011,2012)$ and Singh et al., (2016). In saline soils, availability of micronutrients such as $\mathrm{Fe}, \mathrm{Zn}, \mathrm{Mn}$, and $\mathrm{Cu}$ may either increase or decrease (Moreno et al., 2000). $\mathrm{Na}^{+}$accumulation in plant foliage and their relation with growth in the present investigation are in agreement with the findings of Grieve et al., (2007); GarciaSanchez et al., (2003).

From the observation on the effect of salinity on growth and mineral composition, it may be concluded that in ber specific injury from $\mathrm{Cl}^{-}$ and $\mathrm{Na}^{+}$accumulation in the foliage and nutrient imbalance caused due to high concentration of salinity ions in soil and plant tissues are the chief effects of salinity. Ber cultivars Banarsi Karaka, Narendra Ber Sel.-2 and Ponda can be placed in tolerant group and recommended for commercial cultivation in salt affected areas.

\section{Acknowledgment}

The senior author is thankful to the Dean and Head College of Horticulture and Forestry, NDUAT, Faizabad-224 229, Uttar Pradesh for provide necessary facility to carry out this experiment.

\section{References}

Agrawal, R., Gupta, S., Gupta, N.K., Khandelwal, S.K. and Bhargava, R. 2013. Effect of sodium chloride on gas exchange, antioxidative defense mechanism and ion accumulation in different cultivars of Indian jujube (Ziziphus mauritiana L.). Photosynthetica, 51: 95-101.

Al-Abdoulhadi, I.A., Dinar, H.A., Ebert, G. and Büttner, C. 2012. Influence of salinity levels on nutrient content in leaf, stem and root of major date palm
(Phoenix Dactylifera L) cultivars. Int. Res. J. Agric. Sci. Soil Sci., 2(8): 341346.

Al-Abdoulhadi, I.A., Dinar, H.A., G. Ebert and Büttner, C. 2011. Effect of salinity on leaf growth, leaf injury and biomass production in date palm (Phoenix dactylifera L) cultivars. Indian J. Sci. and Technol. 4(11): 1542-1546.

Al-Absi, K., Qrunfleh, M. and Abu-Sharar, T. 2003. Mechanism of salt tolerance of two olive (Olive europaea L.) cultivars as related to electrolyte concentration and toxicity. Acta Horticult., 618: 281-290.

Arndt, S.K., Wanek, W., Clifford, S.C. and Popp, M. 2000. Contrasting adaptations to drought stress in fieldgrown Ziziphus Mauritiana and Prunus Persica trees: Water relations, osmotic adjustment and carbon isotope composition. Aust. J. Plant Physiol., 27: 985-996.

Arzani, A. 2008. Improving salinity tolerance in crop plants: a biotechnological view. In Vitro Cell. Develop. Biol. Plant, 44: 373-383.

Ashraf, M., Mukhtar, N., Rehman, S. and Rha, E.S. 2004. Salt-induced changes in photosynthetic activity and growth in a potential medicinal plant bishop's weed (Ammi majus L). Photosynthetica, 42: 543-550.

Awasthi, O.P., Pathak, R.K. and Pandey, S.D. 1995. Effect of sodicity and salinity levels on four scion cultivars budded on Indian jujube (Ziziphus mauritiana). Indian J. Agric. Sci., 65(5): 363-367.

Baatour, O., Kaddour, R., Wannes, W.A., Lachaâl, M. and Marzouk, B. 2010. Salt effects on the growth, mineral nutrition, essential oil yield and composition of marjoram (Origanum majorana). Acta Physiol. Plant., 32: 
45-51.

Bolarin, M.C., Perez-Alfocea, F., Cano, E.A., Estan, M.T. and Caro, M. 1993. Growth, fruit yield and ion concentration in tomato genotypes after pre and post emergence salt treatments. J. Am. Soc. Hortic. Sci., 118: 655-660.

Chartzoulakis, K.S. 2005. Salinity and olive: growth, salt tolerance, photosynthesis and yield. Agric. Water Manat., 78: 108-121.

Feigin, A. 1985. Fertilization management of crops irrigated with saline water. Plant Soil, 89, 285-299.

Garcia-Sanchez, F., Carvajal, M., Cerda, A. and Martinez, V. 2003. Response of "Star Ruby" grapefruit on two rootstocks to $\mathrm{NaCl}$ salinity. Journal of Horticultural Science \& Biotechnology, 78(6): 859-865.

Grieve, A. M., Prior, L. D. and Bevington, K. B. 2007. Long term effects of saline irrigation water on growth, yield, and fruit quality of "Valencia" orange trees. J. Agric. Res., 58(4): 342-348.

Hernandez, J.A. and Almansa, M.S. 2002. Short term effects of salt stress on antioxidant systems and leaf water relations of pea leaves. Physiol. Plant., 115: 251-257.

Hokmalipour, S. 2015. Effect of salinity and temperature on seed germination and seed vigor index of chicory (Chichorium intynus L.), cumin (Cuminium cyminium L.) and fennel (Foeniculum vulgare). Indian J. Sci. Technol., 8: 1-9.

Liu, X.N. and Baird, W.V. 2004. Identification of a novel gene, HAABRC5, from Helianthus annuus (Asteraceae) that is upregulated in response to drought, salinity, and abscisic acid. Amer. J. Bot., 91: 184191.
Martinuzzo, R. 2006. Jujube Ziziphus jujuba Mill Rhamnaceae. A Tuscan farm house "the Jujube" Casa Vacanza "I1 Giuggiolo".

Moreno, D.A., Pulgar, G. and Romero, L. 2000. Yield improvement in zucchini under salt stress: Determining micronutrient balance. Sci. Hortic., 86: $175-183$.

Munns, R. 2002. Comparative physiology of salt and water stress. Plant Cell Environ., 25: 239-250.

Munns, R. 2005. Genes and salt tolerance: bringing them together. New Phytol., 167: 645-663.

Overlach, S., Diekmann, W. and Raschke, K. 1993. Phosphate translocator of isolated guard-cell chloroplasts from Pisum sativum L. transports glucose6-phosphate. Plant Physiol., 101: 1201-1207.

Pareek, S. and Yahia, E.M. 2013. Postharvest biology and technology of ber fruit. In: Horticultural Reviews (Editors: Jules Janick). Published by Wiley. Pp. 41: 200-240.

Pradeep, T. and Jumbhale, N.D. 2000. Ploidy level variations for stomata, chloroplast number, pollen size and sterility in ber (Ziziphus mauritiana Lamk). Indian J. Gen., 60: 519-525.

Rao, V.K. and Singh, H.K. 2004. Effect of salinity levels on growth performance of aonla (Emblica officinalis Gaertn.) Prog. Hort. 36(1): 87-90.

Shen, C.Q., Cao, S.Y., Guo, J.Y., Chen, Y.L., Xue, H.B., Si, P., Zhang, L. and Xie, S.X. 2009. Salt tolerance of four jujube cultivars. Acta Hortic., 840: 161-166.

Sherani, J., Jillani, M.S. and Ahmad, T. 2017. Ber (Zizyphus mauritiana L.) production and quality as influenced by different salinity levels in water. Pak. J. Agric. Sci., 4(3):645-652.

Shiyab, S.M., Shatnawi, M.A., Shibli, R.A., 
Al Smeirat, N.G., Ayad, J. and Akash, M.W. 2013. Growth, nutrient acquisition, and physiological responses of hydroponic grown tomato to sodium chloride salt induced stress. J. Plant Nutrition, 36: 665-676.

Shukla, S.K. and Singh, G.N. 1996. Nutrient imbalances in bael (Aegle marmelos Correa) leaves as induced by salinity and sodicity. Indian J. Plant Physiol., 1: 293-294.

Singh, A. and Yadav, R.K. 2017. Nursery management in fruit crops in saltaffected soils. In: Nursery management in fruit crops in saltaffected soils. Publisher: ICARCSSRI, Karnal, Haryana.

Singh, A., Kumar, A., Datta, A. and Yadav, R.K. 2018a. Evaluation of guava (Psidium guajava) and bael (Aegle marmelos) under shallow saline watertable conditions. Indian J. Agric. Sci., 88(5): 720-725.

Singh, A., Sharma, P.C., Kumar, A., Meena, M.D. and Sharma, D.K. 2015. Salinity induced changes in chlorophyll pigments and ionic relations in bael (Aegle marmelos Correa) cultivars. J. Soil Salinity and Water Quality, 7: 404.

Singh, A., Sharma, P.C., Kumar, A., Meena, M.D. and Sharma. D.K. 2018b. Genotypic differences for salt tolerance in bael (Aegle marmelos) cultivars. Indian J. Agric. Sci., 88 (3): 435-441.

Singh, A., Sharma, P.C., Meena, M.D., Kumar, A., Mishra, A.K., Kumar, P., Chaudhari, S.K and Sharma, D.K. 2016. Effect of salinity on gas exchange parameters and ionic relations in bael (Aegle marmelos Correa). Indian J. Hort., 73: 48-53.

Verma, S.S., Verma, R.S., Verma, S.K., Yadav, A.L. and Verma, A.K. 2018. Impact of salt stress on plant establishment, chlorophyll and total free amino acid content of ber (Zizyphus mauritiana Lamk.) cultivars. J. Pharmacognosy and Phytochem., 7(2): 556-559.

\section{How to cite this article:}

Verma, S.S., R.P. Verma, S.K. Verma, A.L. Yadav and Verma, A.K. 2018. Responses of Ber (Zizyphus mauritiana Lamk.) Varieties to Different Level of Salinity. Int.J.Curr.Microbiol.App.Sci. 7(07): 580-591. doi: https://doi.org/10.20546/ijcmas.2018.707.071 